\title{
Composite scaffolds for osteochondral repair obtained by combination of additive manufacturing, leaching processes and hMSC-CM functionalization
}

\author{
Andrés Díaz Lantada ${ }^{a} *$, Hernán Alarcón Iniesta ${ }^{b}$, Josefa Predestinación García-Ruíz ${ }^{b}$ \\ a Product Development Laboratory, Mechanical Engineering \& Manufacturing Department, Universidad Politécnica de Madrid (UPM), c/José Gutiérrez Abascal 2, 28006 Madrid, Spain \\ ${ }^{\mathrm{b}}$ Departamento de Biología Molecular, Universidad Autónoma de Madrid, 28049, Cantoblanco, Madrid, Spain
}

Keywords:

Tissue engineering

Scaffolds for tissue repair

Osteochondral repair

Rapid prototyping

Additive manufacture

Computer-aided design \& engineering

\begin{abstract}
$\mathrm{Abst}$ ract
Articular repair is a relevant and challenging area for the emerging fields of tissue engineering and biofabrication. The need of significant gradients of properties, for the promotion of osteochondral repair, has led to the develop ment of several families of composite biomaterials and scaffolds, using different effective approaches, although a perfect solution has not yet been found. In this study we present the design, modeling, rapid manufacturing and in vitro testing of a composite scaffold aimed at osteochondral repair. The presented composite scaffold stands out for having a functional gradient of density and stiffness in the bony phase, obtained in titanium by means of computer aided design combined with additive manufacture using selective laser sintering. The chondral phase is obtained by sugar leaching, using a PDMS matrix and sugar as porogen, and is joined to the bony phase during the polymerization of PDMS, therefore avoiding the use of supporting adhesives or additional intermediate layers. The mechanical performance of the construct is biomimetic and the stiffness values of the bony and chondral phases can be tuned to the desired applications, by means of controlled modifications of different parameters. A human mesenchymal stem cell (h MSC) conditioned medium (CM) is used for improving scaffold response. Cell culture results provide relevant information regarding the viability of the composite scaffolds used.
\end{abstract}

\section{Introduction}

Tissue engineering is based on the combination of biological, physical and engineering knowledge to promote the artificial development of improved replacements for tissues and organs linked to surgical repair strategies. A very relevant component, involved in tissue engineering processes, is the extra cellular matrix (ECM) or tissue engineering scaffold which serves as the framework for cell growth, aggregation, phenotype expression and final tissue development [1]. According to biomimetic design principles, the biomaterials used as scaffolds should be porous, so as to allow cell migration during the colonization process, as well as the transport of nutrients and waste to and from cells. Such biomaterial constructs also have to be resistant enough to withstand mechanical demands, especially if final implantation is desired. In addition, as cells are capable of feeling their microenvironment and of responding to the substrate texture upon which they lie, by changing their overall morphology, cytoskeleton configuration, and intra and extracellular signaling, an increasing number of studies are focusing on

\footnotetext{
* Corresponding author.

E-mail address: adiaz@etsii.upm.es (A. Díaz Lantada).

URL: http://www.upm.es (A. Díaz Lantada).
}

advanced design and manufacturing technologies, so as to generate and modify the structures and surfaces of biomaterial. Aspects such as porosity, pore distribution and size or surface micro and nano textures promote cell adherence, migration and proliferation within the scaffold, for subsequent gene expression and differentiation into relevant cell types. Hence, both tissue progenitor cells and the extra cellular matrices play a fundamental role in tissue engineering strate gies. The controlled design and fabrication of biomaterials used as scaffold structures are becoming increasingly important for regenerative medicine [2 4].

Most processes for manufacturing micro porous structures for tissue engineering [5] involve a combination of materials in some step of the process and a final phase separation or leaching process, for obtaining a solid part with distributed small pores. Among most extended pro cesses, gas assisted injection molding is an industrial method based on injecting a molten resin or thermoplastic into a mold cavity and then applying a quantity of pressurized gas into the resin, so as to help to fill out the mold cavity and to create hollows and pores in the poly mer. The incorporation of foaming agents as additives to polymers also allows the manufacture of polymeric parts with pores. In many strategies including tissue strategies, to obtain 3D porous structures is absolutely required to irrigate the tissue and maintain an adequate 
liquid dynamics. The use of porogens is also commonplace; normally, the process involves mixing a liquid prepolymer with solid particles (typically wax, sugar, salt...). Once polymerization is produced, normally by UV exposure or by heating, a solid structure, formed by a polymeric network with dispersed particles, is obtained. Final porous structure is obtained by dissolving such disperse particles in water, other solvents or by heating. The use of prepolymer water emulsions is also typical for obtaining a polymerizable mixture that after thermal or UV based polymerization provides a polymeric network with pores according to initial water content (i.e. polyHIPEs).

Main alternatives, for improving the control of scaffolds' pore size and distribution, from the design stage, is the use of micro additive manufacturing technologies (AMT), normally working on layer by layer processes, following the geometries obtained with the help of computer aided designs [6 7]. Electro spinning can be also adapted to "layer by layer" fabrication and used for obtaining 3D porous structures [8], even though the process is not as repetitive as the use of micro AMT. The progressive increase in the precision of additive manufacturing technologies, together with their improved versatility thanks to a continuously increasing set of materials available for layer by layer processing, is greatly promoting applications linked to micro and even nano manufacturing of complex 3D geometries for very innova tive medical solutions in several fields [5].

Scaffolds with design controlled structures have been obtained by means of rapid prototyping technologies including: selective laser sintering (SLS) [9], layered hydrospinning [10], laser stereolithography (SLA) [11], digital light projection (DLP) [12] or two photon polymeri zation (2PP) [13], and different materials including hydrogels [14], gelatin [7], titanium alloys [15 16], (bio)photopolymers [17] and ceramics [18]. However in vitro validation of rapid prototyped scaffolds is not commonplace, as most combinations of processes and materials do not provide adequate biomaterials and in many cases generate toxic components. Nevertheless some highly interesting research has already been published, including in vitro validation and systematic toxicity assessment [19 20]. Advances in the field of biopolymers [19 21] together with the possibilities provided by thin coatings [11], are bringing new possibilities to this area, although access to such mate rials and technologies is not always easy, as some of them are currently under development or only available in large research centers.

In any case, it seems clear that a universal methodology for tissue engineering scaffold development is not yet available, first of all due to the complexity of biological materials and systems, but also due to all the possible design resources, manufacturing technologies and related materials available, whose results have not been systematically compared. For instance, additive manufacturing technologies allow precise control of final geometries from the design stage; however such designs are normally obtained by combining Euclidean based (simple) geometries and final result does not mimic adequately the complexity of biomaterials. On the other hand, scaffolds obtained by phase separation and more "traditional" processes typically lead to more biomimetic sponges, even though their final outer form and repeatability are more difficult to control, than using computer aided strategies linked to rapid prototyping using additive processes.

Therefore, further research is needed to address the advantages of combining different technologies [22] for manufacturing enhanced, even personalized, scaffolds for tissue engineering studies and extra cellular matrices with global (outer) geometries defined as implants for tissue repair. In addition, increasing data shows that progenitor cell niche formation is absolutely needed for tissue development and repair [23]. Indeed, the niche composition and 3 D structure play an important role in stem cell state and fate. The niche is created by the specific combination of trophic factors produced by progenitor cells to maintain the capability for tissue repair and regeneration and by a specific extracellular matrix. Recent studies have helped to highlight the extreme relevance of the incorporation of adequate growth factors, within the scaffold, for promoting biological regulation, cell differentiation, angiogenesis and final tissue viability [24 26]. Such inclusion of biochemical effects, derived from the incorporation of growth factors, adds additional uncertainties to the already complex to understand interactions between scaffolds' structure, morphology and mechanical properties. Consequently, studies addressing the syner gies between ECMs and growth factors and their impact on tissue viability are needed, in the quest for a general methodology for tissue engineering scaffold development.

The aforementioned tissue engineering challenges are even greater in applications aimed at articular repair, where several types of tissues (bones, cartilages, ligaments, tendons...) must be replaced, if possible using a single multifunctional scaffold capable of promoting cell adhe sion, growth, migration and differentiation into different types of tis sues. The need of relevant gradients of properties for the promotion of osteochondral repair has led to the development of composite scaffolds using different approaches previously reviewed [27,28]. Typical methods for the manufacture of scaffolds with functional gradients of properties include: the use of embedded (nano ) fibers and textiles within polymeric matrixes $[27,28,29]$, the combination of rigid lattice structures with cell carrying hydrogels $[27,28,30]$, the use of multi layered constructs [31,32] (normally requiring adhesives within layers), and computer aided tissue engineering constructs [33]. Among the most promising approaches, towards stable and effective composite scaffolds, it is important to note the combination of: a) phase separation or leaching processes, normally for obtaining the soft chondral phase, with b) computer aided rapid prototyping technologies based on addi tive manufacturing, usually for manufacturing the rigid bony phase [34]. In spite of the very positive results shown by metallic rapid prototyped prostheses and scaffolds for bone repair [35], most successful composite scaffolds for osteochondral repair are based on polymer ceramic com posites [31,34,36], polymer polymer composites [37], ceramic ceramic composites [38], ceramic metal composites [39] and metal metal composites [40]. Interestingly, metal polymer composites, which could benefit from the stiffness of metals for the bony phase and from the elasticity of polymers for the chondral phase are not so common.

In this study we present the design, modeling, rapid manufacturing and in vitro testing of a composite scaffold aimed at osteochondral repair. The presented composite scaffold stands out for having a func tional gradient of density and stiffness in the bony phase, which is obtained in titanium by means of computer aided design combined with additive manufacture using selective laser sintering. The chondral phase is achieved by sugar leaching, using a PDMS matrix and sugar as porogen, and is joined to the bony phase during the polymerization of PDMS, therefore avoiding the use of supporting adhesives or additional intermediate layers. The mechanical performance of the construct is biomimetic and the stiffness values of the bony and chondral phases can be tuned to the desired applications, by means of controlled modi fications of the computer aided designs, of the materials used, of the rate of porogen employed, among other options that promote the versa tility of the proposed approach. Cell culture results, carried out using $\mathrm{h}$ MSCs with the help of growth factors generated by the own progeni tor stem cells, provide relevant information regarding the viability of the composite scaffolds used and help us to plan forthcoming research activities. The following section details the materials and methods used, before describing and analyzing main results and proposing future steps towards the concept of the universal scaffold for osteochondral repair.

\section{Materials and methods}

\subsection{Computer aided design of functionally graded lattice and porous structures}

Computer aided design of the different geometries was carried out with the help of NX 8.5 (Siemens PLM Solutions), mainly using combinations of parametric and matrix based features and Boolean 
operations. Fig. 1 shows three lattices and porous structures for sub sequent solid freeform fabrication of the bony phases of biomimetic composite scaffolds for potential osteochondral repair strategies. In the three designs included, density and stiffness vary along their thickness, as happens in real tissues. The spatial control of scaffolds' properties is important, for making cells grow in a similar environment as can be found in real organisms and for artificially obtaining final tissues with a biomimetic structure. For instance, in bone tissue engi neering, the transition between the trabecular and cortical regions is interesting. Therefore, scaffolds designed in a similar fashion as those from Fig. 1, may prove useful in articular repair strategies, especially for the bony phase. The three scaffolds count with 10 to 12 layers of pores or lattices and their density changes linearly from one layer to the following one, by using confluent trusses, conical elements and spherical pores. Their overall size is $20 \times 20 \times 20 \mathrm{~mm}^{3}$. The distances between pores and lattices are designed according and taking into ac count the manufacturing precision of state of the art rapid prototyping facilities working with parts in the $\mathrm{mm}^{3}$ range.

\subsection{Combination of additive and leaching processes towards composite scaffolds}

The bony (more rigid) phase is obtained by automated rapid proto typing using additive manufacturing technologies working after the CAD models. Preliminary prototypes for geometrical validation of the lattice structures are obtained using the "SLA 3500" laser stereolithography system from 3D Systems (3D Systems, 333 Three D
Systems Circle, Rock Hill, SC 29730, USA), after format conversion to .stl and batch preparation with the help of 3D Lightyear software. The material used for these preliminary prototypes is a photoreactive epoxy resin with trade name "Accura ${ }^{\circledR}$ 60" from 3D Systems. The prototyping process by stereolithography can be briefly described as follows. Laser stereolithography is an additive manufacturing process using a vat of liquid UV curable photopolymer or "resin" and a UV laser to build parts layer by layer in an additive way. On each layer, the laser beam traces a cross section pattern on the surface of the resin. Exposure to the UV laser cures (and solidifies) the pattern traced on the resin, adhering it to the previous layer. After a pattern has been traced, the machine elevator platform moves vertically a single layer of thickness, typically $0.05 \mathrm{~mm}$ to $0.15 \mathrm{~mm}$. Then, a resin filled blade sweeps across the partial cross section, re coating it with fresh material. On this new liquid surface, the subsequent layer pattern is traced, adhering to the previous layer. A complete 3D part is formed by repeated iterations of this process. After manufacture, parts are cleaned by immersion in a chemical bath (normally $510 \mathrm{~min}$ in isopropylic alcohol or acetone) and finally post cured to improve mechanical properties in a UV oven for around $1020 \mathrm{~min}$, depending on the resin's specifications.

The degree of precision and the quality of surface finish attainable by additive photo polymerization processes are remarkable: i.e. layer thicknesses down to $25 \mu \mathrm{m}$ and wall thicknesses down to $0.6 \mathrm{~mm}$ for parts with volumes in the $\mathrm{mm}^{3}$ range and details even reaching $500 \mathrm{~nm}$ for parts in the $\mu^{3}$ range. However, most resins available are not adequate for in vitro or in vivo tests in contact with cells or living organisms, as some debris may be toxic. In consequence, for the cell

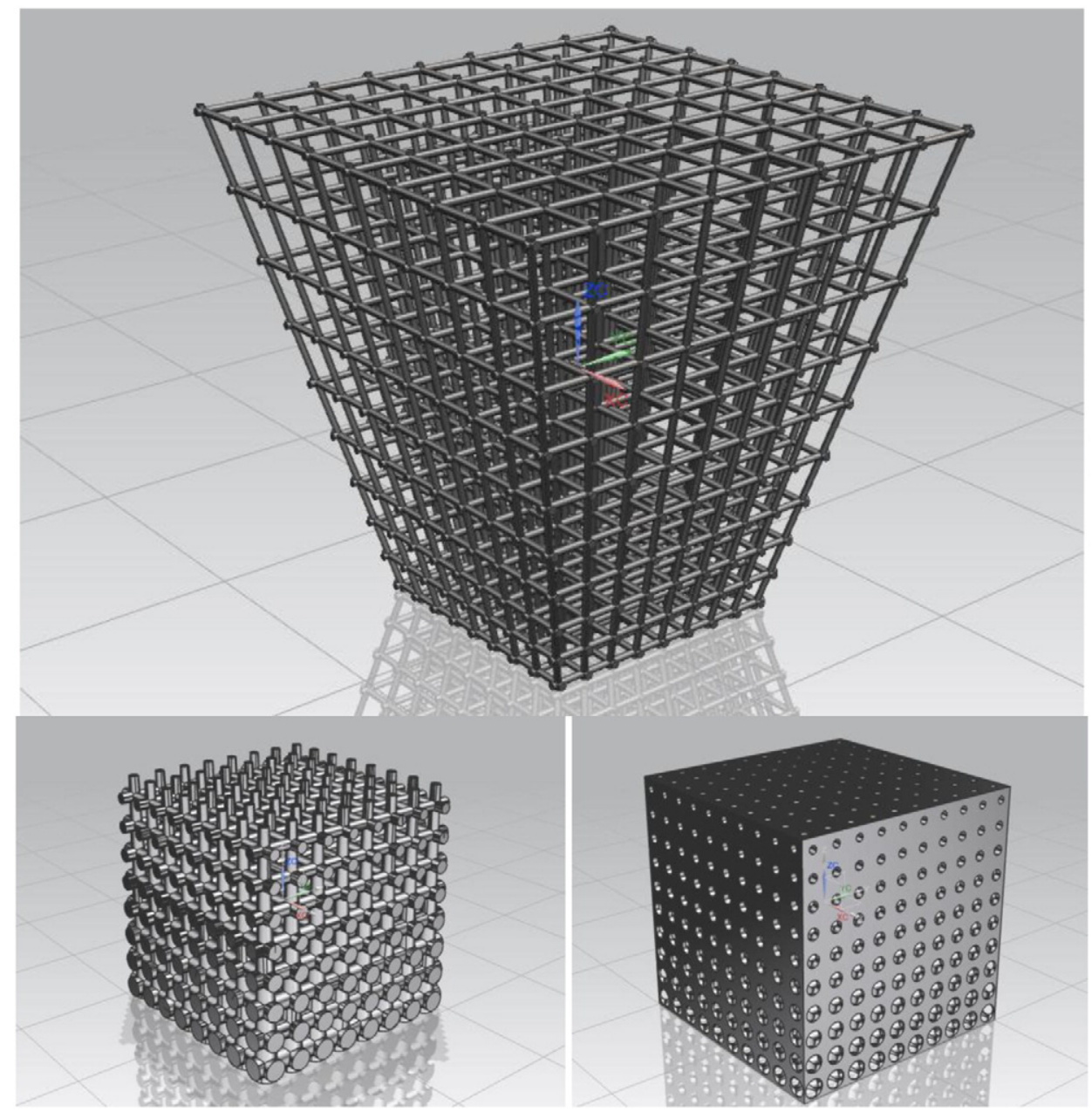

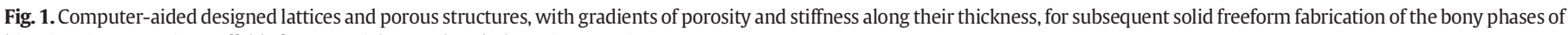
biomimetic composite scaffolds for potential osteochondral repair strategies. 
culture trials we obtain additional prototypes in titanium by state of the art selective laser sintering. In selective laser sintering the models are also printed layer by layer, by a laser that draws thin lines upon tita nium powder. The laser melts and bonds the powder, so as to form a thin layer of the model. After a layer is printed, a new layer of fresh powder is spread over the surface by a roller. The printer has a print chamber that is heated to just below the melting point of the powder and the laser beam adds the extra energy to melt the powder, forming a solid model. For obtaining the prototypes in titanium for the bony phase we have contracted the services of the 3D Print Lab of iMaterialise (http://i.materialise.com/).

The chondral (more flexible) phase is obtained by a sugar leaching process and includes several simple steps detailed further on and shown schematically in Fig. 2. First of all, the Neukasil RTV 20 pre polymer and the Neukasil A2 cross linker are mixed and degasified, following manufacturer's instructions. Subsequently, the PDMS sugar mixture is obtained with the help of a Taurus vertical mixer blender spinning at $1200 \mathrm{rpm}$ during $1 \mathrm{~min}$ just before casting. We use a mixture of $50 \% 50 \%$ of PDMS sugar in weight for obtaining a very flexible support for the chondral phase. The mixture is casted into a rapid mold, where the Ti lattice has been previously introduced, until the lower layers of the lattice are embedded with the PDMS sugar mixture.
A rapid prototyped insert can be also placed into the mold for modify ing the surface of the chondral phase, for example for obtaining vertical pores aimed at the promotion of vascularization, as proposed elsewhere [41]. The construct is left at room temperature during $24 \mathrm{~h}$ for adequate polymerization. Once the PDMS mixtures are polymerized, de molding is accomplished for obtaining the desired composite scaffolds, after adequate cutting and leaching. Particle leaching, for the desired phase separation, is achieved by water immersion and systematic squeezing of the chondral phase. Final drying leads to the desired implants, which are shown in Section 3.

\subsection{FEM based simulations applied to the composite scaffolds for osteochondral repair}

The different geometries are simulated using the finite element method capabilities of NX 8.5 (Siemens PLM Solutions) for studying the mechanical performance of the different scaffolds designed and for analyzing potential applications in tissue repair. Main details, regarding material, mesh, loads, boundary conditions, solver param eters used and post processing analyses, are described under these lines.

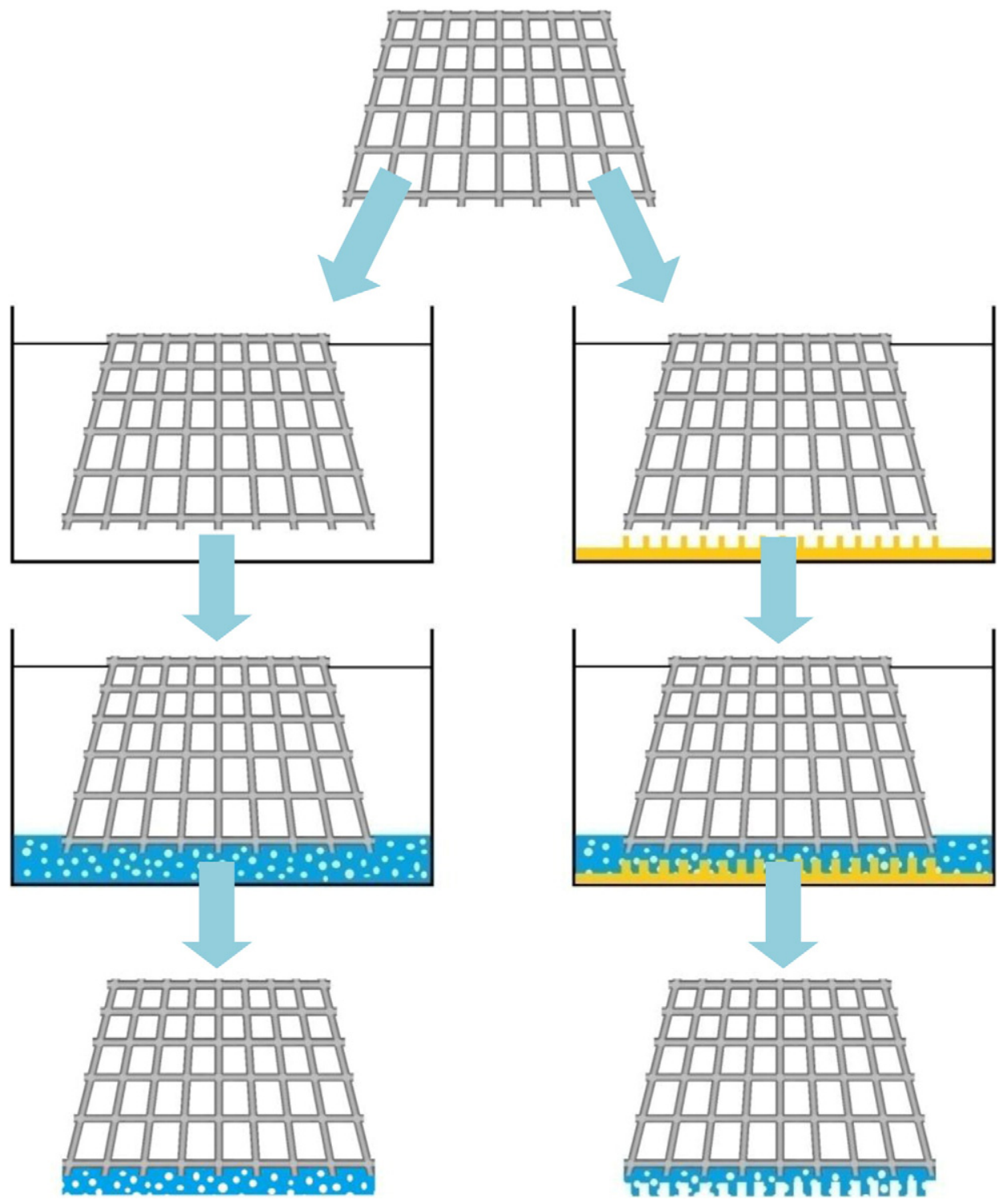

Fig. 2. Scheme of the manufacturing process used for obtaining the porous PDMS chondral phase connected to the Ti lattice of the bony phase, hence leading to a composite scaffold. 


\subsection{Material and mesh}

Tetrahedral 10 node elements are used for meshing, which is carried out with the help of the automatic meshing and refine tool from the software employed, which provides elements with sizes below $0.25 \mathrm{~mm}$ in almost all cases. Additionally, more than $85 \%$ of the elements obtained had a skewness value lower than 0.7 , which provides adequate meshes for the purposes of the present study. Titanium alloy, with mechanical properties similar to those of the alloy used for addi tive manufacture of the metallic lattice structure, has been applied to the bony phase of the composite scaffold. A soft polymer, defined with mechanical properties like those of the PDMS used for the softer layer of the composite scaffold, has been applied to the chondral phase, which has been modeled using NX 8.5 as a block with pores of $450 \mu \mathrm{m}$, similar to the mean diameter of the sugar particles used as porogens in the sugar leaching process.

Titanium alloy bulk properties include a density of $4450 \mathrm{~kg} / \mathrm{m}^{3}$, a Young modulus of $117 \mathrm{GPa}$, a Poisson ratio of 0.33 and a yield strength of $760 \mathrm{MPa}$, properties similar to those of the polymers subsequently used for prototype manufacture. The soft polymer resembling the PDMS has the following bulk properties: a density of $1200 \mathrm{~kg} / \mathrm{m}^{3}$, a Young modulus of $2.45 \mathrm{MPa}$ (according to a Shore A 50 polymer), a Poisson ratio of 0.38 and a yield strength of $15 \mathrm{MPa}$.

\subsection{Boundary conditions}

Loads are applied as a group of punctual forces along " $z$ " direction on the upper faces and edges of the different structures, trying to promote symmetry and homogeneous loading. A load of $500 \mathrm{~N}$ has been vertically ( $-\mathrm{z}$ axis) applied to the upper surface of the chondral phase. The obtained stresses are below the material's yield strength, so we can assume linearity for the subsequent post processing. As boundary conditions, the displacements of the lower faces and edges of the metallic lattice are fixed. Contacts between the metallic lattice and the PDMS sponge are automatically generated and perfect gluing is applied as boundary condition.

\subsection{Solver parameters and post processing}

From the different possibilities of NX 8.5, for carrying out FEM simulations, "NX Nastran" solver and "structural analysis" type (solu tion type SESTATIC 101) are selected with the option of "element itera tive solver" activated, as 3D elements are used for the simulations. Simulations are performed at a default ambient temperature of $25^{\circ} \mathrm{C}$. It is important to remark the compatibility between the design and simulation programs, as geometries can be directly imported for simu lation, without the typical limitations of universal format (.igs, .stl, .stp...) conversions and the information loss they normally involve.

Once the simulations are carried out, post processing tools allow for a straightforward measurement of displacements for subsequent calculation of the equivalent Young moduli of the different parts of the composite scaffold, as further discussed in Section 3.2. Details regarding the results from the simulations developed are presented, summarized and discussed in Section 3.

\subsection{Cell culture process for in vitro validation}

The hMSCs used in this work were isolated in a Percoll gradient from 1 or $2 \mathrm{ml}$ of human bone marrow samples from anonymous healthy donors and provided by hematology services of the Hospital La Princesa, the Jiménez Díaz Foundation and the University Biobank of Málaga, and expanded as described previously $[42,43]$. Cells were plated and incubated using DMEM L plus 10\% FBS of selected batches. Cells were collected by treatment with $0.25 \%$ trypsin EDTA. Cell culture mediums were prepared by the research services of Molecular Biology Center "Severo Ochoa" (CSIC UAM).
For the preparation of CM hMSC we used 810 p100 culture plates at $80 \%$ confluence for each batch. Cells were washed with PBS and incu bated in DMEM L starved of FBS and supplemented with $2 \mathrm{mM}$ pyru vate during $24 \mathrm{~h}$. Afterwards culture medium was collected, cleaned of any floating cell by centrifugation at $1500 \mathrm{rpm}$ in a bench centrifuge during $5 \mathrm{~min}$. The clean supernatant was cooled down on ice during $30 \mathrm{~min}$, centrifuged in a Sorvall to remove salt precipitations and kept in $2 \mathrm{ml}$ aliquots at $-30^{\circ} \mathrm{C}$ until use. We avoid any samples with repeated freeze thaw.

The lattice scaffolds were UV irradiated, individually placed in $25 \mathrm{ml}$ Falcon tubes and received the following treatment: i) wash using $0.5 \mathrm{ml}$ $\mathrm{PBS}$ and 5 min centrifugation; ii) treatment with $2 \mathrm{M}$ acetic acid during 20 min, rapid neutralization and PBS wash; iii) treatment with hMSC CM during $24 \mathrm{~h}$ or DMEM LG as control; and iv) scaffold seed with 150,000 hMSC and incubation in DMEM L 10\% FBS during $48 \mathrm{~h}$ at $37^{\circ} \mathrm{C}$ in $5 \% \mathrm{CO}_{2}$ as early indicated $[44,45]$. Then, the lattice scaffolds were cut into smaller slices and pieces, individually placed in M24 tissue dishes, rinsed with ice cold PBS and fixed in 3.7\% formaldehyde in PBS during 30 min at RT and washed in PBS. Afterwards, samples were washed with PBS and fixed in 3.7\% formaldehyde in PBS during $30 \mathrm{~min}$ and processed for immunofluorescence [46,47]. To this end, cells were permeated and all cell soluble proteins were removed by incubation with $0.5 \%$ Triton in a CSK buffer containing $10 \mathrm{mM}$ pipes, pH 6.8, $3 \mathrm{mM} \mathrm{MgCl}_{2}, 100 \mathrm{mM} \mathrm{NaCl}, 1 \mathrm{mM}$ EGTA, $0.3 \mathrm{M}$ sucrose for $30 \mathrm{~min}$ on ice. After the treatment, samples were cleaned and fixed with 3.7\% formaldehyde and equilibrated in PBS. Nuclei were stained with DAPI (CALBIOCHEM) and observed using an inverted IX81 Olympus coupled with a DP72 digital camera. Cytoskeleton was observed by determining $\alpha$ Tubulin (1:2000, Sigma) and a mouse derived secondary antibody labeled with Alexa 488 (1:500, Invitrogen) for finally address ing the energetic behavior of the cells and their viability upon the scaffolds.

\section{Results and discussion}

\subsection{Validation of the design and manufacturing process}

Fig. 3 shows different prototypes of lattice structures with function ally graded physical properties, according to the confluent truss based design of Fig. 1. Fig. 3a shows a preliminary prototype intended for geometrical validation obtained by laser stereolithography in epoxy resin. Fig. 3b shows a couple of lattices obtained in titanium by selective laser sintering, as previously detailed, ready for the incorporation of the porous PDMS sponge for final cell culture trials. In the epoxy prototypes the minimal truss diameter is $0.5 \mathrm{~mm}$, while in the titanium ones the minimal dimension is $\mathrm{d}=0.7 \mathrm{~mm}$. The precision of the photopolymerization based technologies is higher than that of the selective laser sintering or selective laser melting processes, but the commercially available photo polymers used typically prevent ade quate cell culture. Some interesting comparisons between these tech nologies, taking account of precision, available materials, including examples of bio photopolymers, and focusing on cell culture matrices have been published [48 49].

Fig. 4 shows a couple of rapid prototypes of the complete composite scaffolds, including a Ti lattice structure, obtained by additive manu facture, and an embedded porous PDMS sponge, obtained by a sugar leaching process. The remarkable elasticity of the chondral phase can be appreciated in the lower image, in which the prototype can be seen gently compressed by a polymeric spatula. The range of pore sizes of the chondral phase is between 60 and $250 \mu \mathrm{m}$ in diameter, the smaller pores consequence of trapped air bubbles and the larger ones conse quence of sugar leaching, showing results similar to previous studies [44]. It is important to note that the PDMS completely embeds two layers of the titanium lattice, thus avoiding the use of intermediate connecting elements or adhesives. The process can be almost directly industrialized for mass production, thanks to the improvements in 


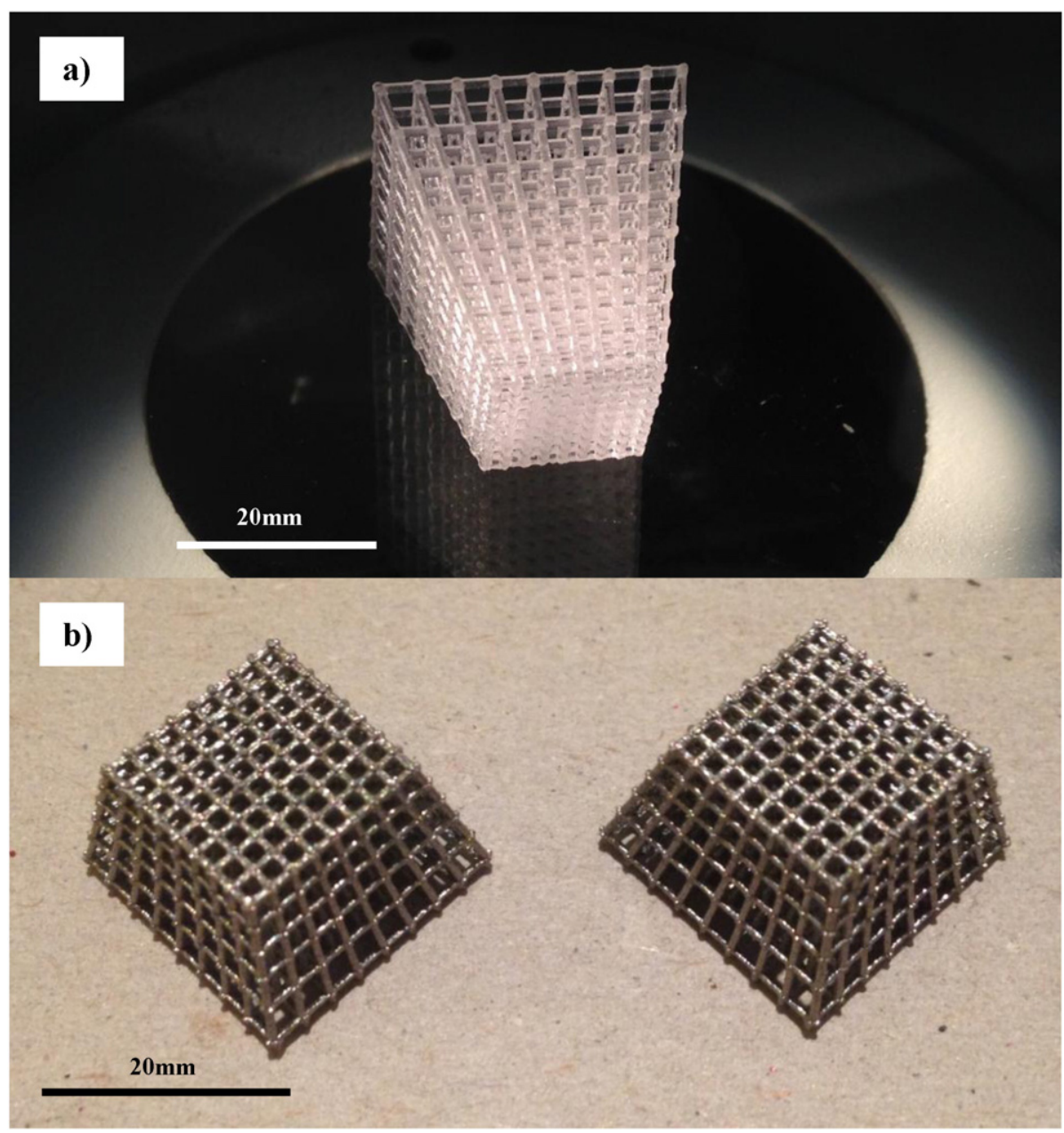

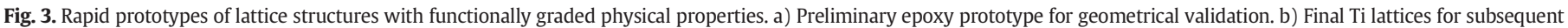
infiltration of PDMS and final cell culture trials.

manufacturing speed of 3D printers and to the simple sugar leaching process used for incorporating the flexible chondral layer. Several materials can be combined, as additive manufacture works with poly mers, ceramics, alloys and composites, for adjusting the stiffness of the different phases of the scaffold.

\subsection{Mechanical performance of the composite scaffolds}

Fig. 5a shows, schematically, the finite element model of the com posite scaffold for osteochondral repair, including the main boundary conditions, connections between bodies and loads used, according to the explanation provided in Section 2.3. Simulation solutions are also summarized in Fig. 5b and c, which respectively detail the obtained displacement and stress fields. The displacement and stress fields are drawn upon the deformed structure, showing the deformation in abso lute magnitude. The applied force of $500 \mathrm{~N}$ upon the upper surface of the chondral phase $\left(15 \times 15 \mathrm{~mm}^{2}\right.$ in the simulated construct) is similar to a pressure applied of $2 \mathrm{MPa}$ and mimics the conditions that the construct should withstand, once implanted. Under such pressure, the chondral phase deforms importantly (around a 90\%, which can be clearly seen in the figures), as should happen in vivo, being the cartilage a shock absorber. The stress values are limited in the chondral phase to around $10 \mathrm{MPa}$, clearly below the PDMS limit used for the prototypes. The bony titanium phase deforms only a $0.03 \%$ and its stress values reach $135 \mathrm{MPa}$, also well below the Ti limit. Convergence analyses were carried out using both the $\mathrm{h}$ method and the $\mathrm{p}$ method. In the first case, mesh was refined down to $0.05 \mathrm{~mm}$ size elements, thus adding more elements for checking results with improved accuracy. In the second case, the complexity of the shape functions was analyzed by using tetrahedral 4 node and 10 node elements. Using both approaches the changes noticed, when comparing the displacement and stress results obtained, were lower than $4 \%$. In consequence, we consider the obtained results accurate enough for design purposes. These results can be used for evaluating the equivalent Young moduli of the bony and chondral phases, for additional comparison with the biomaterials to be substituted. Simulation results lead to a Young modulus of the chondral phase of around $2 \mathrm{MPa}$, which corresponds to the typical order of magnitude of cartilage moduli measured in previous experi ences [50] and to characterization results from our initial studies linked to the manufacture of porous PDMS [44]. The Young modulus of the titanium lattice varies gradually from $2.7 \mathrm{GPa}$ to $6.6 \mathrm{GPa}$, which is also in the range of the transitions from trabecular to cortical bone, which the lattice would aim to replace or repair. In consequence, according to our results, the mechanical performance of the construct is bio mimetic, as proposed in seminal papers in the field [51], and the stiffness values of the bony and chondral phases can be tuned to the desired applications, by means of controlled modifications of the computer aided designs, of the materials used, of the rate of porogen employed, among other options that promote the versatility of the proposed approach. The design parameters used for these first 


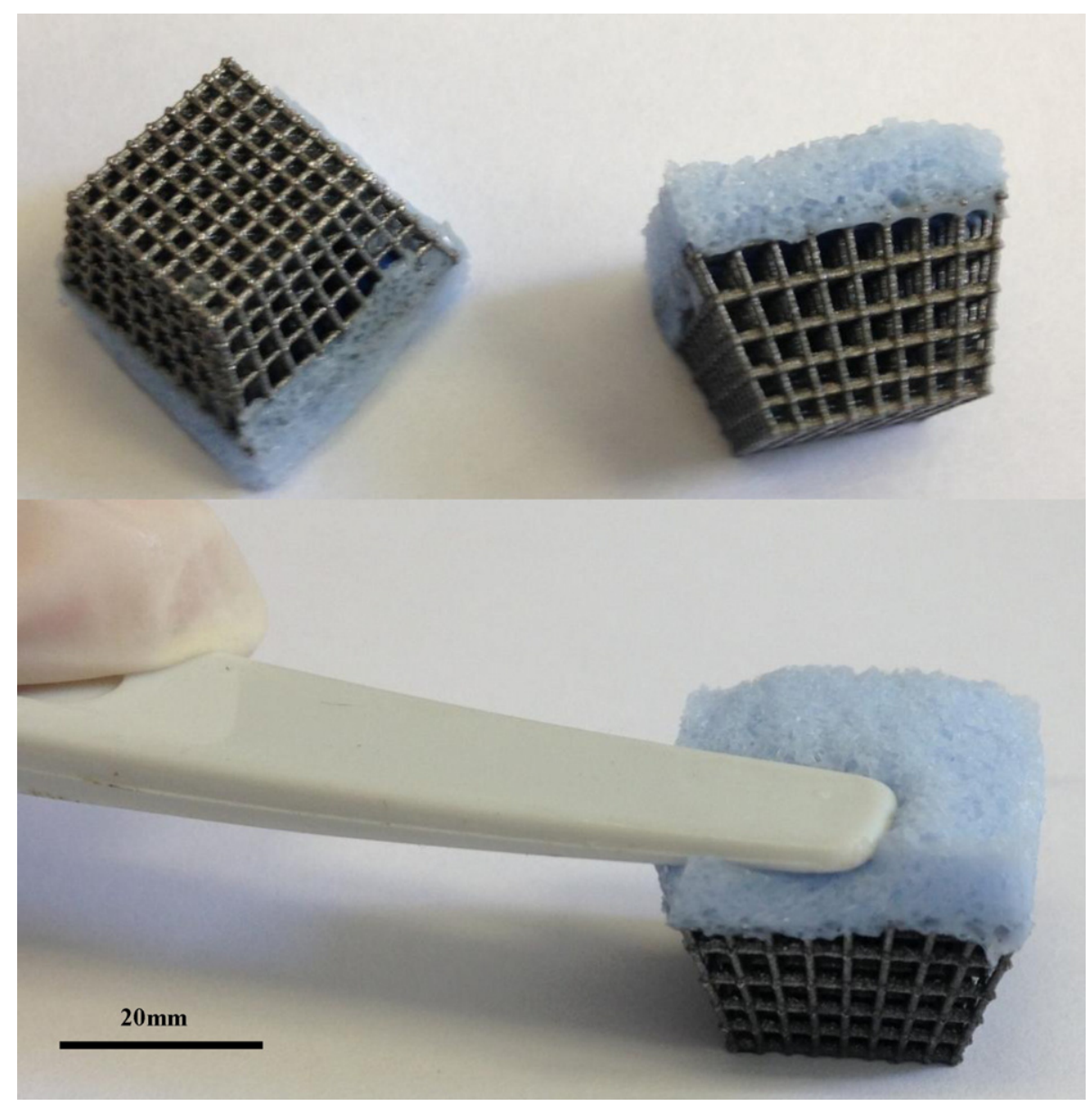

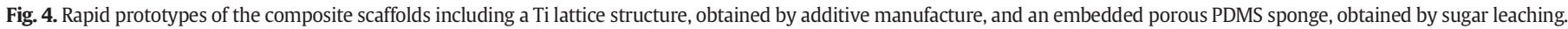
The remarkable elasticity of the chondral phase can be appreciated in the lower image.

prototypes provide results in the expected ranges of mechanical proper ties of the biomaterials to be repaired or replaced.

\subsection{Cell culture results for in vitro validation}

Regarding cell culture trials, some results from hMSCs interacting with the composite Ti PDMS scaffold are shown in the four images of Fig. 6. To this, end composites were exposed to a conditioned medium produced by hMSC as reported in early work, then seeded with cells and incubated with DMEM low glucose and 10\% FBS during $48 \mathrm{~h}$. The interface between the Ti lattice and the PDMS network is shown. Fig. $6 a$ and $b$ focuses more on a zone of the bony phase, as can be seen by the presence of holes with crossing trusses at almost $90^{\circ}$, while Fig. $6 \mathrm{c}$ and $\mathrm{d}$ focuses more on the more circular pores of the chondral phase. The nuclei are stained in blue (DAPI) and their rounded forms without blebs indicating that the cells are in healthy conditions and well adhered to the porous scaffold. It is important to note that more than 50 cells $/ \mathrm{mm}^{2}$ can be appreciated. Several spherical ellipsoidal cell aggregations can be appreciated, with potential application for promoting differentiation into osteocytes for bone repair, as part of the global osteochondral repair strategy. Thus, CM hMSC and composite scaffolds are sponges with excellent porosity for hMSC to adhere.

In order to assess the energetic behavior of cells a second set of trials was carried out, seeding again both phases of the scaffold, specially focusing on the interface between the Ti lattice and the porous PDMS and labeling both the nuclei and the cytoskeletons, as previously detailed in the experimental section. Fig. 6e and f helps to show the good energetic behavior of the cells, both in the bony and in the chondral phase. Fig. 6e shows several cells attached and spreading upon a Ti truss of the bony phase and Fig. 6 f shows the cells attached, spreading and forming bridges within and upon the PDMS porous network of the chondral phase. More than 40 expanded active cells can be appreciated per visual field in the bony phase and more than 50 in the chondral phase. Additional studies will be realized using improved confocal microscopy facilities and performing osteochondral differentiation as realized using porous PDMS and may let us provide more details about the three dimensional configuration of the cells within the whole construct, but we understand that the provided validation shows promising results.

Our results show that the cells and the composites scaffolds are excellent companions for potential tissue repair strategies. In case of tissue damage, the hMSCs seeded lattice and porous scaffolds may con stitute a functionally graded support, in order to allow the permeation of nutrients and debris, to promote oxygenation, to enable adaptation and to provide cellular communication systems, capable of locally inhibiting the immune system and of activating tissue repair, following the fluid dynamic, although additional assessments, both in vitro and in vivo, need to be performed.

Last but not the least, the hMSCs seeded composite scaffolds offer interesting possibilities to study cellular mechanisms present in differ ent types of tissues, specially the interactions between bone and carti lage, although they may well be useful for studying other types of musculoskeletal tissues. The cell material interactions may be extend ed to a triad composed by hMSCs composite scaffold endoderm/ exoderm derived cells for studies on complex tissues.

Regarding future studies, it would be interesting to further study cell behavior using biomimetic constructs with functional gradients of properties. The computer aided lattice designs presented here may be 

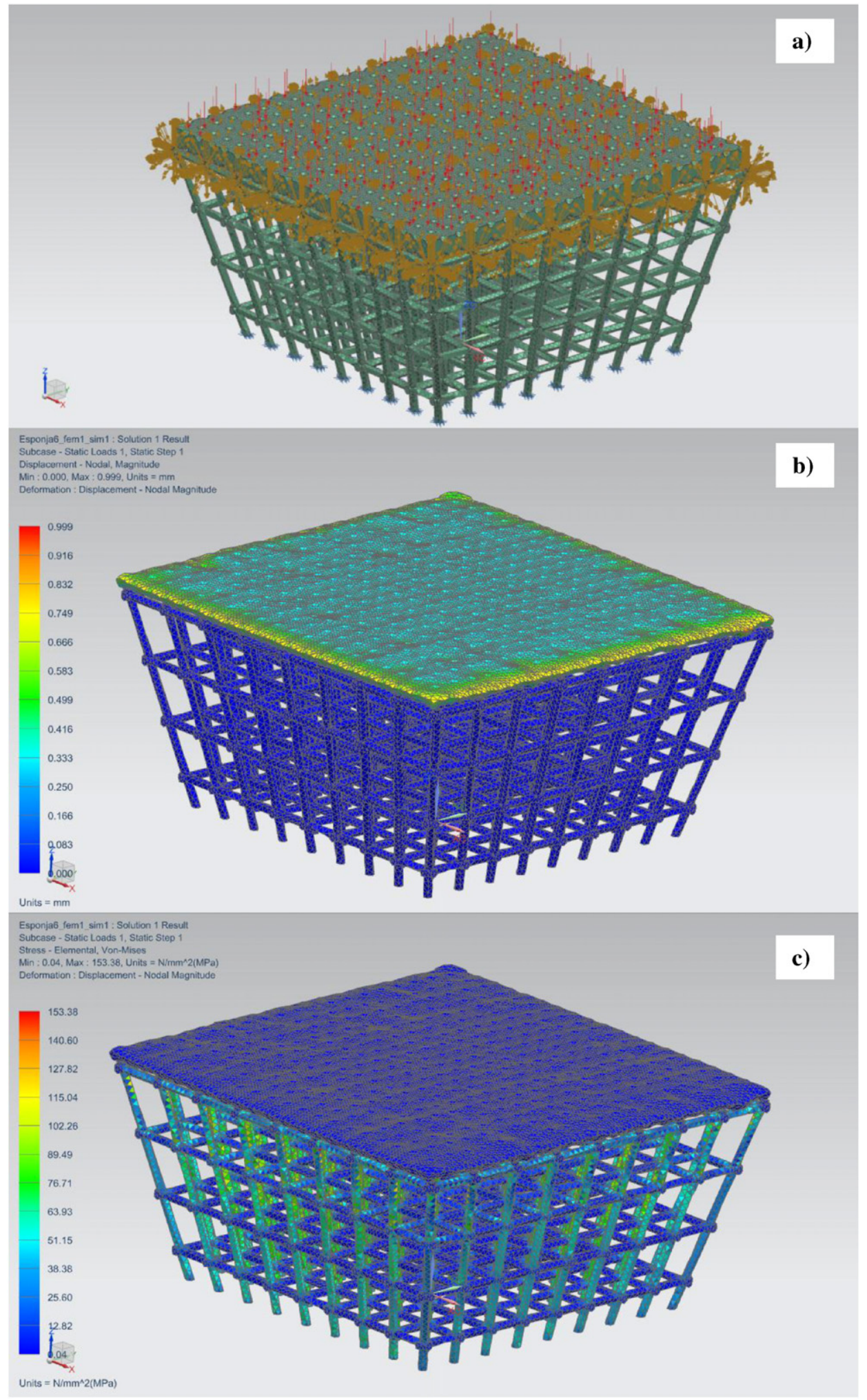

Fig. 5. Finite-element model of the composite scaffold for osteochondral repair (a) and simulation solutions for the displacement (b) and stress (c) fields.

improved, in terms of bone biomimicry, by using recently proposed strategies based on the use of multi morphology transition hybridiza tion CAD design of minimal surface porous structures [52]. The irregular features of living tissues can be also taken into account and modeled by means of multi scale approaches [53] and by resorting to stochastic design procedures [54]. (For interpretation of the references to color in this figure legend, the reader is referred to the web version of this article.)

\section{Conclusions}

In this study we have presented the design, modeling, rapid manufacturing and in vitro testing of a composite scaffold aimed at osteochondral repair. The presented composite scaffold stands out for having a functional gradient of density and stiffness in the bony phase, which has been obtained in titanium by means of computer aided 


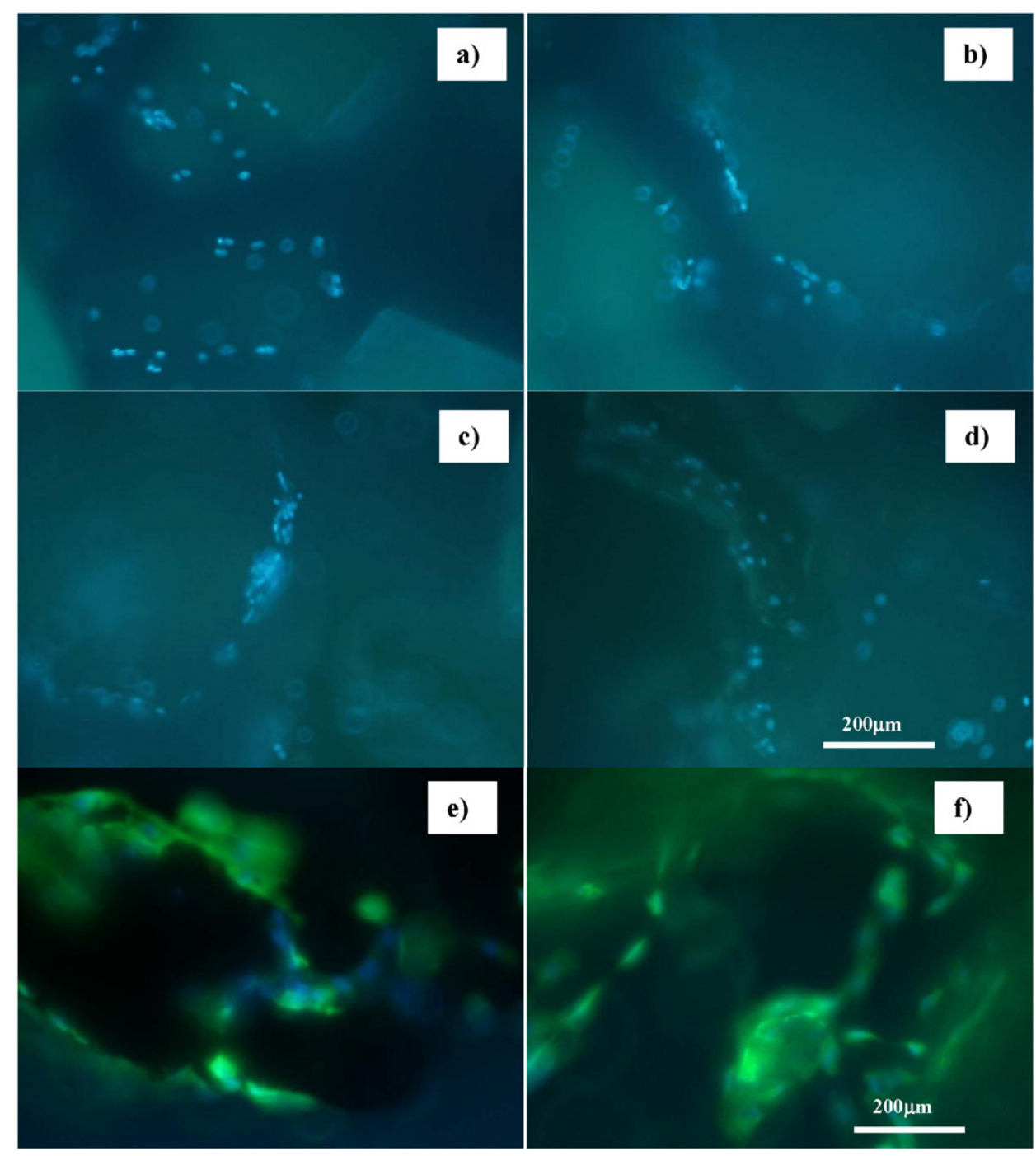

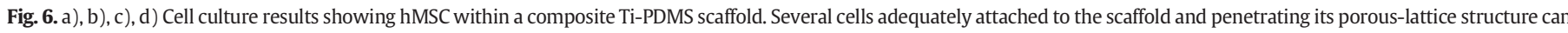

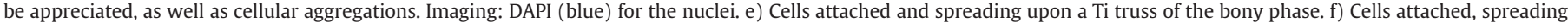

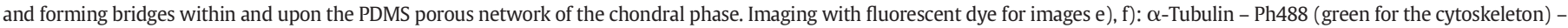
DAPI (blue for the nuclei). The same scale has been used for all images.

design combined with additive manufacture using selective laser sintering. The chondral phase has been manufactured by sugar leaching, using a PDMS matrix and sugar as porogen, and is joined to the bony phase during the polymerization of PDMS, therefore avoiding the use of supporting adhesives or additional intermediate layers. The mechanical performance of the construct is biomimetic and the stiffness values of the bony and chondral phases can be tuned to the desired applications, by means of controlled modifications of the computer aided designs, of the materials used, of the rate of porogen employed, among other options that promote the versatility of the proposed approach. Cell culture results, carried out using h MSCs with the help of growth factors generated by the own progenitor stem cells, have provided relevant information regarding the viability of the composite scaffolds used and have helped us to plan forthcoming research activi ties. Even though the final construct is not biodegradable, it may consti tute a feasible device for studying osteochondral repair processes in vitro and, in the future, similar designs and combinations of materials may serve as advances prostheses for large bone repair, in which bio degradability is not always needed. The generated designs are at the disposal of colleagues who may find them useful for further collabora tive research in the fields of tissue engineering and biofabrication.

\section{Acknowledgments}

We acknowledge Mr. Pedro Ortego García, from the Product Devel opment Lab at TU Madrid, for his continued support with prototyping trials. We acknowledge Mr. Antonio Sillero Martínez, from the Machine Engineering Division at TU Madrid, for his help with computer aided design tasks. We acknowledge reviewers for their proposals for improvement, which have helped us to achieve a much more detailed final version. Their selection of interesting references made us also aware of interesting alternatives to the processes used here.

\section{References}

[1] R. Langer, J.P. Vacanti, Tissue engineering, Science 260 (1993) 920.

[2] W.E. Thomas, D.E. Discher, V.P. Shastri, Mechanical regulation of cells by materials and tissues, MRS Bull. 35 (2010) 578

[3] W.L. Chen, M. Likhitpanichkul, A. Ho, C.A. Simmons, Integration of statistical modeling and high-content microscopy to systematically investigate cell-substrate interactions, Biomaterials 31 (2010) 2489

[4] A. Buxboim, D.E. Discher, Stem cells feel the difference, Nat. Methods 7 (9) (2010) 695

[5] A. Díaz Lantada, Handbook of Advanced Design and Manufacturing Technologies for Biodevices, Springer Verlag, 2013. 
[6] P.J.S. Bartolo, H. Almeida, T. Laoui, Rapid prototyping and manufacturing for tissue engineering scaffolds, Int. J. Comput. Appl. Technol. 36 (2009) 1.

[7] J.Y. Tan, C.K. Chua, K.F. Leong, Indirect fabrication of gelatin scaffolds using rapid prototyping technology, Virtual Phys. Prototyping 5 (1) (2010) 45.

[8] A.K. Ekaputra, Y. Zhou, S.M.K. Cool, D.M. Hutmacher, Composite electrospun scaffolds for engineering tubular bone grafts, Tissue Eng. A 15 (12) (2009) 3779.

[9] S. Lohfeld, M.A. Tyndyk, S. Cahill, N. Flaherty, V. Barron, P.E. Mc Hugh, A method to fabricate small features on scaffolds for tissue engineering via selective laser sintering, J. Biomed. Sci. Eng. 3 (2010) 138.

[10] R. Tzezana, E. Zussman, S.A. Levenberg, Ultra-porous scaffold for tissue engineering, created via a hydrospinning method, Tissue Eng. Part C 14 (4) (2008) 281.

[11] A. Díaz Lantada, A. Mosquera, J.L. Endrino, P. Lafont, Design and rapid prototyping of DLC coated fractal surfaces for tissue engineering applications, J. Appl. Phys. Conf. Ser. 252 (2010) 012003.

[12] J. Stampfl, H. Fouad, S. Seidler, R. Liska, R.F. Schwanger, A. Woesz, P. Fratzl, Fabrication and moulding of cellular materials by rapid prototyping, Int. J. Mater. Prod. Technol. 21 (4) (2004) 285.

[13] R. Infür, N. Pucher, C. Heller, H. Lichtenegger, R. Liska, V. Schmidt, L. Kuna, A. Haase, ] Stampfl, Functional polymers by two-photon 3D lithography, Appl. Surf. Sci. 254 (2007) 836.

[14] P.S. Maher, R.P. Keatch, K. Donnelly, Z. Paxton, Formed 3D Bio-scaffolds Via Rapid Prototyping Technology, Presented at 4th European Conference of the IFMBE, IFMBE Proceedings, 22(17) 2009, p. 2200.

[15] G.E. Ryan, A.S. Pandit, D. Apatsidis, Porous titanium scaffolds fabricated using a rapid prototyping and powder metallurgy technique, Biomaterials 29 (2008) 3625.

[16] P.H. Warnke, T. Douglas, P. Wollny, E. Sherry, M. Steiner, S. Galonska, S.T. Becker, I.N. Springer, J. Wiltfang, S. Sivananthan, Rapid prototyping: porous titanium alloy scaffolds produced by selective laser melting for bone tissue engineering, Tissue Eng Part C Methods 15 (2) (2009) 115.

[17] J. Stampfl, S. Baudis, C. Heller, R. Liska, A. Neumeister, R. Kling, A. Ostendorf, M. Spitzbart, Photopolymers with tunable mechanical properties processed by laserbased high-resolution stereolitoraphy, J. Micromech. Microeng. 18 (2008) 125014

[18] S.C. Cox, J.A. Thornby, G.J. Gibbons, M.A. Williams, K.K. Mallick, 3D printing of porous hydroxyapatite scaffolds intended for use in bone tissue engineering applications, Mater. Sci. Eng. C 47 (2015) 237-247.

[19] M. Schuster, C. Turecek, B. Kaiser, J. Stampfl, R. Liska, F. Varga, Evaluation of biocompatible photopolymers. I: photoreactivity and mechanical properties of reactive diluents, J. Macromol. Sci. A 44 (2007) 547.

[20] M. Schuster, C. Turecek, B. Kaiser, J. Stampfl, R. Liska, F. Varga, Evaluation of biocompatible photopolymers II: Further reactive diluents, Monatsh. Chem. 138 (2007) 261

[21] F. Jung, C. Wischke, A. Lendlein, Multifunctional cardiovascular implants: challenges and hurdles, MRS Bull. 35 (2010) 607.

[22] J.Y. Tan, C.K. Chua, K.F. Leong, Fabrication of channeled scaffolds with ordered array of micro-pores through microsphere leaching and indirect rapid prototyping technique, Biomed. Microdevices 15 (2013) 83.

[23] C.K.F. Chan, C.C. Chen, C.A. Luppen, D.L. Kraft, J.B. Kim, A. De Boer, K. Wei, J.A. Helms, C.J. Kuo, I.L. Weissman, Endochondral ossification is required for hematopoietic stem cell niche formation, Nature 457 (7228) (2009) 490.

[24] T.P. Richardson, M.C. Peters, A.B. Ennett, D.J. Mooney, Polymeric system for dual growth factor delivery, Nat. Biotechnol. 19 (2001) 1029.

[25] A. Perets, Y. Baruch, F. Weisbuch, G. Shoshany, V. Neufeld, S. Cohen, Enhancing the vascularization of three-dimensional porous alginate scaffolds by incorporating controlled release basic fibroblast growth factor microspheres, J. Biomed. Mater. Res. A 65 (4) (2003) 489.

[26] M.W. Laschke, M. Rücker, G. Jensen, C. Carvalho, R. Mülhaupt, N.C. Gellrich, M.D. Menger, Incorporation of growth factor containing Matrigel promotes vascularization of porous PLGA scaffolds, J. Biomed. Mater. Res. A 85 (2) (2008) 397.

[27] F.T. Moutos, F. Guilak, Composite scaffolds for cartilage tissue engineering, Biorheology 45 (3-4) (2008) 501-512.

[28] P. Nooeaid, V. Salih, J.P. Beier, A.R. Boccaccini, Osteochondral tissue engineering: scaffolds, stem cells and applications, J. Cell. Mol. Med. 16 (10) (2012) 2247-2270

[29] E. Kon, A. Mutini, E. Arcangeli, M. Delcogliano, G. Filardo, N.N. Aldini, D. Pressato, R Quarto, S. Zaffagnini, M. Marcacci, Novel nanostructured scaffold for osteochondra regeneration: pilot study in horses, J. Tissue Eng. Regen. Med. (2009)http://dx.doi. org/10.1002/term.243.

[30] E.J. Caterson, L.J. Nesti, W.J. Li, K.G. Danielson, T.J. Albert, A.R. Vaccaro, R.S. Tuan, Three-dimensional cartilage formation by bone marrow-derived cells seeded ion polylactide/alginate amalgam, J. Biomed. Mater. Res. 57 (2001) 394-403.
[31] J.K. Sheerwood, S.L. Riley, R. Palazzolo, S.C. Brown, D.C. Monkhouse, M. Coates, L.G. Griffith, L.K. Landeen, A. Ratcliffe, A three-dimensional osteochondral composite scaffold for articular cartilage repair, Biomaterials 23 (2008) 4739-4751.

[32] R.M. Schek, J.M. Taboas, S.J. Hollister, P.H. Krebsbach, Tissue engineering osteochondral implants for temporomandibular joint repair, Orthod. Craniofacial Res. 8 (2005) 313-319.

[33] W. Sun, B. Starly, J. Nam, A. Darling, Bio-CAD modeling and its application in computer-aided tissue engineering Comput Aided Des. 37 (11) (2005) 1097-1114.

[34] H. Da, S.-J. Jia, G.-L. Meng, J.-H. Cheng, W. Zhou, Z. Xiong, Y.-J. Mu, J. Liu, The impact of compact layer on osteochondral tissue engineering, PLoS ONE 8 (1) (2013), e54838.

[35] K. Alvarez, H. Nakajima, Metallic scaffolds for bone regeneration, Materials 2 (2009) $790-832$.

[36] X. Zhang, W. Chang, P. Lee, Y. Wang, M. Yang, J. Li, S.G. Kumbar, X. Yu, Polymer-ceramic spiral structured scaffolds for bone tissue engineering: effect of hydroxyapatite, composition on human fetal osteoblasts, PLoS ONE (2014) 9(1).

[37] H.-W. Kang, J.H. Park, T.-Y. Kang, Y.-J. Seol, D.-W. Cho, Unit cell-based computeraided manufacturing system for tissue engineering, Biofabrication 4 (2012) 015055.

[38] Y. Hakamatsuka, H. Irie. Prosthetic artificial bone having ceramic layers of different porosity. US Patent 5152791 A, 1989.

[39] C. Zhao, H. Zhang, B. Cai, G. Wang, H. Fan, X. Zhang, Preparation of porous PLGA/Ti biphasic scaffold and osteochondral defect repair, Biomater. Sci. 1 (7) (2013) 703-710.

[40] M.P. Staiger, A.M. Pietak, J. Huadmai, G. Diaz, Magnesium and its alloys as orthopedic biomaterials: a review, Biomaterials 27 (9) (2006) 1728-1734.

[41] H.C.S. Ko, B.K. Milthorpe, C.D. Mc Farland, Engineering thick tissues - the vascularization problem, Eur. Cell. Mater. 14 (2007) 1-19.

[42] D.P. Lennon, S.E. Haynesworth, S.P. Bruder, N. Jaiswal, A.I. Caplan, Human and animal mesenchymal progenitor cells from bone marrow: identification of serum for optimal selection and proliferation, In vitro Cell. Dev. Biol. 32 (2006) 602

[43] S. Ogueta, J. Muñoz, E. Obregon, E. Delgado-Baeza, J.P. García-Ruiz, Prolactin is a component of the human sinovial liquid and modulates the growth and chondrogenic differentiation of bone marrow-derived mesenchymal stem cells, Mol. Cell. Endocrinol. 190 (1-2) (2002) 51.

[44] A. Díaz Lantada, H. Alarcón Iniesta, B. Pareja Sánchez, J.P. García-Ruíz, Free-form rapid-prototyped PDMS scaffolds incorporating growth factors promote chondrogenesis, Adv. Mater. Sci. Eng. (2014), 612976.

[45] M.F. Pittenger, A.M. Mackay, S.C. Beck, R.K. Jaiswal, R. Douglas, J.D. Mosca, M.A. Moorman, D.W. Simonetti, S. Craig, D.R. Marshak, Multilineage potential of adult human mesenchymal stem cells, Science 284 (5411) (1999) 143.

[46] M. Romero-Prado, C. Blázquez, C. Rodríguez-Navas, J. Muñoz, I. Guerrero, E. Delgado-Baeza, J.P. García-Ruiz, Functional characterization of human mesenchymal stem cells that maintain osteochondral fates, J. Cell. Biochem. 98 (2006) 1457.

[47] A. Javed, B. Guo, S. Hiebert, J.Y. Choi, J. Green, S.C. Zhao, M.A. Osborne, S. Stifani, J.L. Stein, J.B. Lian, A.J. van Wijnen, G.S. Stein, Groucho/TLE/R-esp proteins associate with the nuclear matrix and repress RUNX (CBF (alpha)/AML/PEBP2(alpha)) dependent activation of tissue-specific gene transcription, J. Cell Sci. 113 (Pt 12) (2000) 2221.

[48] A. Ovsianikov, V. Mironov, J. Stampfl, R. Liska, Engineering 3D cell-culture matrices: multiphoton processing technologies for biological \& tissue engineering applications, Expert Rev. Med. Devices 9 (6) (2012) 613-633.

[49] A. Díaz Lantada, B. Pareja Sánchez, C. Gómez Murillo, J. Urbieta Sotillo, Fractals in tissue engineering: towards biomimetic cell-culture matrices, microsystems and microstructured implants, Expert Rev. Med. Devices 10 (5) (2013) 629-648.

[50] J.M. Mansour, Biomechanics of Cartilage, in: C.A. Oatis (Ed.), Kinesiology: The Mechanics and Pathomechanics of Human Movement, Lippincott Williams and Wilkins, 2003.

[51] D.W. Hutmacher, Scaffolds in tissue engineering and bone cartilage, Biomaterials 21 (24) (2000) 2529-2543.

[52] N. Yang, Z. Quan, D. Zhang, Y. Tian, Multi-morphology transition hybridization CAD design of minimal surface porous structures for use in tissue engineering, Comput. Aided Des. 56 (2014) 11-21.

[53] N. Yang, K. Zhou, Effective method for multi-scale gradient porous scaffold design and fabrication, Mater. Sci. Eng. C 43 (1) (2014) 502-505.

[54] N. Yang, L. Gao, K. Zhou, Simple method to generate and fabricate stochastic porous scaffolds, Mater. Sci. Eng. C 56 (1) (2015) 444-450. 